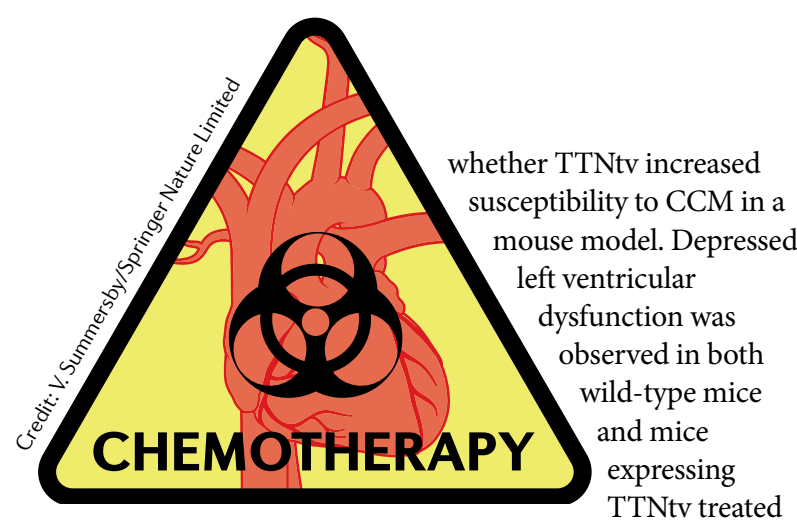

with anthracycline. However, wild-

type mice recovered to baseline levels by week 8 , whereas left ventricular dysfunction persisted through to week 12 in the mice expressing TTNtv.

In summary, the increased

The increased

burden of rare

variants among

patients

with $\mathrm{CCM}$

suggests that

genetics are

an important

determinant

in CCM

susceptibility

burden of rare variants among

patients with CCM suggests

that genetics are an important determinant in CCM susceptibility and outcomes. "The identification of genetic risk factors opens new opportunities to identify cancer patients at high risk of CCM," conclude the investigators.

Karina Huynh

ORIGINAL ARTICLE Garcia-Pavia, P. et al. Genetic variants associated with cancer therapy-induced cardiomyopathy. Circulation https://doi.org/ 10.1161/CIRCULATIONAHA.118.037934 (2019)

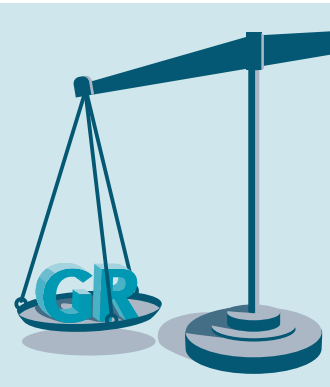

Credit: S. Bradbrook/ Springer Nature Limited

46

alterations

that favour more GR signalling and less MR

signalling are cardioprotective

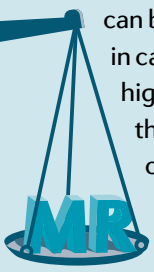

can become deleterious in cardiomyocytes and highlight the critical role of the opposing regulation of GR and MR signalling.

Currently, patients with heart failure are treated with drugs

that block MR signalling. "Our findings suggest that development of a single drug that functions as an antagonist for MRs and an agonist for GRs might represent an improved therapeutic approach for treating heart disease," remark Oakley and Cidlowski. "Development of a single drug that targets both GRs and MRs should be possible because the two receptors are structurally related," they add. The researchers note that the study was performed in male mice, and explain that future studies should assess $G R$ and MR signalling in the hearts of female mice.

\section{Irene Fernández-Ruiz}

ORIGINAL ARTICLE Oakley, R. H. et al.

Cardiomyocyte glucocorticoid and mineralocorticoid receptors directly and antagonistically regulate heart disease in mice. Sci. Signal. 12, eaau9685 (2019)
DYSLIPIDAEMIA

\section{Targeting ATP citrate lyase to reduce LDL cholesterol}

Although the use of lipid-lowering drugs such as statins has resulted in a substantial decline in cardiovascular events, statin therapy alone is often insufficient for reducing plasma LDLcholesterol levels to below target values. Two

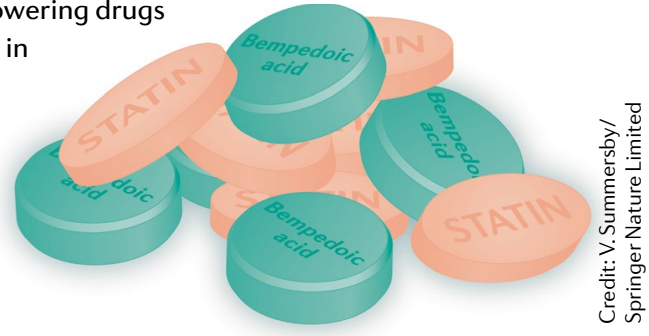
papers published in the NEJM investigated whether the pharmacological or genetic inhibition of ATP citrate lyase, a key enzyme in the cholesterol biosynthesis pathway, can reduce plasma LDL-cholesterol levels without increasing adverse events.

Bempedoic acid has been shown to reduce cholesterol synthesis through inhibition of ATP citrate lyase. Investigators of the CLEAR Harmony randomized, phase III trial sought to examine the efficacy and safety of bempedoic acid therapy added to maximally tolerated statins for 1 year in patients with atherosclerosis and/or familial hypercholesterolaemia.

In total, 1,488 patients were assigned to receive bempedoic acid and 742 to placebo. LDL-cholesterol levels were lower in the bempedoic acid group than in the placebo group at both week 12 (difference -18.1 percentage points, $95 \% \mathrm{Cl}-20.0$ to $-16.1, P<0.001$ ) and at week 24 (difference -16.1 percentage points, $95 \% \mathrm{Cl}-18.2$ to $-14.0, P<0.001)$. The incidence of adverse events was similar in the two treatment groups; however, the percentage of patients who discontinued treatment owing to a reported adverse event was higher among those receiving bempedoic acid than placebo ( $10.9 \%$ versus $7.1 \%)$.

"The present trial provides substantial evidence that bempedoic acid as an adjunct to guideline-based statin regimens appeared to have an acceptable safety profile," conclude the authors of the paper. Two studies on bempedoic acid are ongoing: a long-term, open-label extension of the CLEAR Harmony trial to assess safety data on longer-term exposure and a cardiovascular outcomes study.

In an accompanying article, Ference and colleagues performed a Mendelian randomization study to predict the clinical effect of lowering plasma LDL-cholesterol levels through inhibition of ATP citrate lyase. This enzyme lies upstream of 3-hydroxy-3-methylglutaryl-coenzyme A reductase (HMGCR), the target for statins, in the cholesterol biosynthesis pathway, but whether lowering LDL-cholesterol levels by inhibiting ATP citrate lyase will reduce the risk of cardiovascular events to the same extent as HMGCR inhibition with statins is unclear.

The researchers constructed genetic scores comprising independently inherited variants in ACLY (encoding ATP citrate lyase) or in HMGCR, to mimic the effects of ATP citrate lyase inhibitors and HMGCR inhibitors, respectively. A total of 654,783 individuals, including 105,429 who had a major cardiovascular event, were included in the analysis. For each $10 \mathrm{mg} / \mathrm{dl}$ decrease in plasma LDL-cholesterol level, the ACLY and HMGCR scores showed a similar degree of reduction in the risk of major cardiovascular events. Neither score was associated with increased risk of cancer. "The results of our study thus confirm the mechanism by which ATP citrate lyase inhibition lowers plasma LDL cholesterol levels," remark the authors. "They provide validation for ATP citrate lyase inhibition as a genetic target."

Karina Huynh

ORIGINAL ARTICLES Ray, K. K. et al. Safety and efficacy of bempedoic acid to reduce LDL cholesterol. N. Engl.J. Med. 380, 1022-1032 (2019) | Ference, B. A. et al. Mendelian randomization study of ACLY and cardiovascular disease. N. Engl.J. Med. 380, 1033-1042 (2019)

FURTHER READING Adhyaru, B. B. et al. Safety and efficacy of statin therapy. Nat. Rev. Cardiol. 15, 757-769 (2018) 